\title{
NUEVOS REGISTROS DE LA LAGARTIJA INTRODUCIDA ANOLIS SAGREI (SQUAMATA: DACTYLOIDAE) EN EL ESTADO DE VERACRUZ, MÉXICO
}

\section{NEW RECORDS OF THE INTRODUCED LIZARD ANOLIS SAGREI (SOUAMATA: DACTYLOIDAE) IN THE STATE OF VERACRUZ, MEXICO}

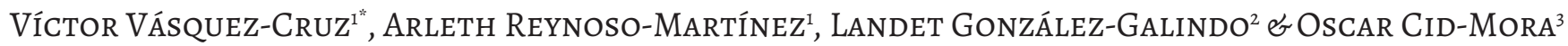

${ }^{1}$ PIMVS Herpetario Palancoatl, Avenida 19 número 5525, Colonia Nueva Esperanza, Córdoba, Veracruz, México.

${ }^{2}$ Providencia, Cuichapa, Veracruz, México.

${ }^{3}$ Centro de Estudios Geográficos, Biológicos y comunitarios, S. C. (Geobicom), Calle Santa María 13, U. Hab. San Román, Códoba, Veracruz, 94542, México.

Correspondence: : victorbiolvc@gmail.com

Received: 2020-12-16. Accepted: 2021-03-29.

Abstract.- Anolis sagrei is a native species from Bahamas, the Cuba Archipelago, and Little Cayman that has been introduced in different parts of the world. This species has been recorded in several locations of Mexico, including state of Veracruz. Herein, we report three new localities for this species that complement the knowledge of its distribution in the state of Veracruz.

Keywords.- Cuban brown anole, Dactyloidae, expansion of the distribution range, introduced species.

Resumen.- Anolis sagrei es una especie nativa de Bahamas, el archipiélago de Cuba y Pequeño Caimán que se ha introducido en diferentes partes del mundo. Esta especie ha sido registrada en varios lugares de México, incluido el estado de Veracruz. Aquí, reportamos tres nuevas localidades para esta especie que complementan el conocimiento de su distribución en el estado de Veracruz.

Palabras clave.- Anolis pardo cubano, Dactyloidae, especies introducidas, expansión de distribución.

Anolis sagrei (Duméril \& Bibron, 1837) es una especie originaria de Cuba, de Las Bahamas e islas aledañas (Powell \& Henderson, 2012). En México es considerada invasora y a nuestro conocimiento, los primeros registros ocurrieron en los estados de Campeche, Quintana Roo y Yucatán por Smith (1938). Posteriormente se ha registrado en los estados de Chiapas, Jalisco, Tabasco, Tamaulipas, Veracruz, Campeche, Quintana Roo y Yucatán, siendo estos últimos tres los que poseen el $70 \%$ de los registros (Vásquez-Cruz et al., 2020). En los últimos 20 años los registros de la especie se han extendido por el Golfo de México hacia los estados de Tabasco y Veracruz, principalmente en áreas urbanas (Vásquez-Cruz et al., 2020). Particularmente, en el estado de Veracruz se ha reportado formalmente en siete municipios: Boca del Rio, Yanga, Alvarado, Córdoba, Banderilla, Los Tuxtlas y Minatitlán (Vásquez-Cruz et al., 2020).

Figure 1. Individuals of Anolis sagrei (LACM PC 2668) from the municipality of Veracruz, Mexico. A) Individuals fighting. B) Individuals exhibiting push up behavior.

Figura 1. Individuos de Anolis sagrei (LACM PC 2668) del municipio de Veracruz, México. A) Individuos combatiendo. B) Individuos exhibiendo comportamiento de flexión.

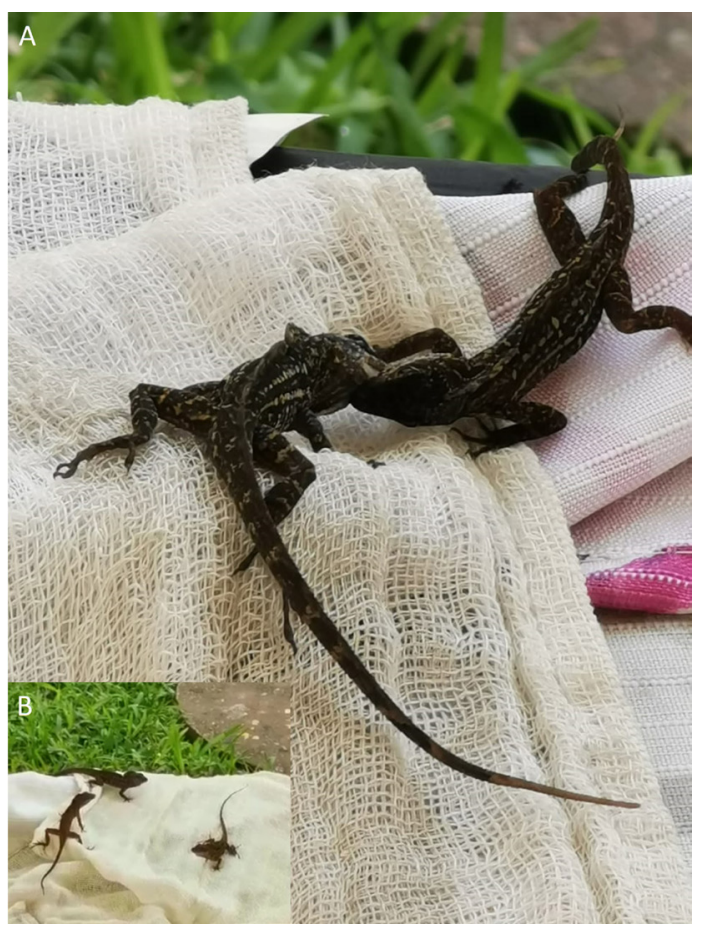




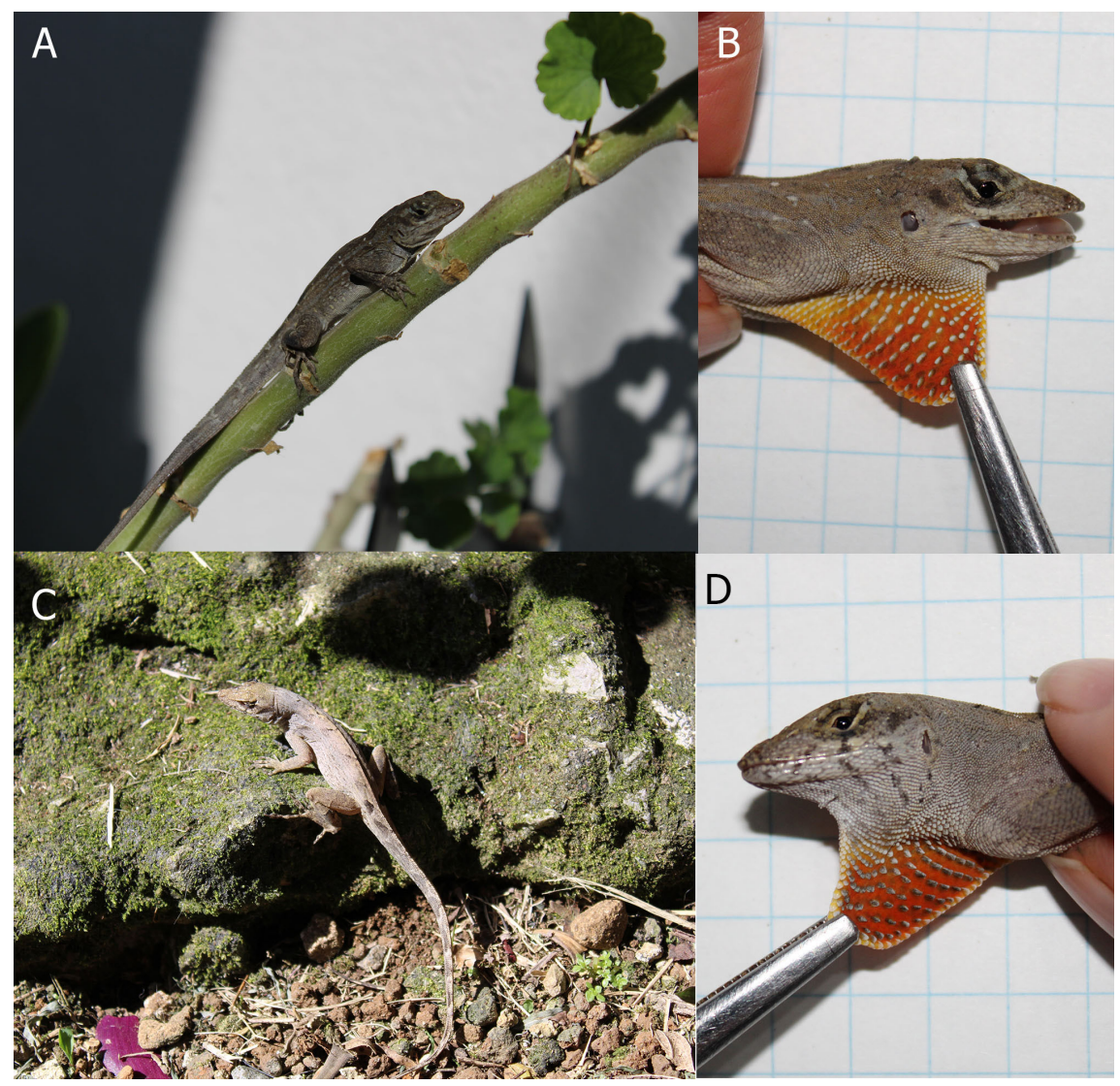

Figure 2. Individuals of Anolis sagrei from the municipality of Cuichapa, Mexico. (A, B) A male individual adult with voucher LACM PC 2680 and $(C, D)$ a male individual with voucher LACM PC 2681.

Figura 2. Individuos de Anolis sagrei del municipio de Cuichapa, México. (A, B) Individuo adulto macho con vales LACM PC 2680 y (C, D) un individuo macho con voucher LACM PC 2681.

Aquí, presentamos tres nuevos registros municipales para A. sagrei en el estado de Veracruz (Tabla 1). Estos registros son resultado de observaciones casuales. Depositamos vales fotográficos digitales en el Natural History Museum of Los Angeles County (LACM PC). Identificamos A. sagrei con base en tres caracteres de diagnóstico: cola comprimida lateralmente, abanico gular anaranjada a roja con un margen amarillo y las escamas de la cola (incluidas las subcaudales) son más puntiagudos y fuertemente quilla (Norval et al., 2002; Köhler, 2008).

El 31 de julio del 2020, alrededor de las 1300 horas, observamos a tres individuos machos de la especie A. sagrei en el interior de un jardín privado localizado en el municipio de Veracruz

Table 1. Previous and current records of the distribution of Anolis sagrei in Veracruz, Mexico. / Tabla 1. Registros previos y actuales de la distribución de Anolis sagrei en Veracruz, México.

\begin{tabular}{|c|c|c|c|}
\hline Municipio & Localidad & Coordenadas & Referencia/Voucher \\
\hline Alvarado & Colonia Los Aguacates & $18.77747^{\circ} \mathrm{N}, 95.769^{\circ} \mathrm{W}$ & Vásquez-Cruz et al., 2020 \\
\hline Alvarado & Mandinga & $19.01960^{\circ} \mathrm{N}, 96.08539^{\circ} \mathrm{W}$ & Toscano-Flores y Calzada-Arciniega 2015 \\
\hline Banderilla & Esquilón, entre Banderillas y Jilotepec & $19.60833^{\circ} \mathrm{N}, 96.975^{\circ} \mathrm{W}$ & MZFC 5479 \\
\hline Boca del Rio & Colonia Las Vegas & $19.13850^{\circ} \mathrm{N}, 96.13130^{\circ} \mathrm{W}$ & Vásquez-Cruz et al., 2020 \\
\hline Córdoba & Córdoba & $18.88317^{\circ} \mathrm{N}, 96.92417^{\circ} \mathrm{W}$ & Venerozo-Tlazalo et al. 2017 \\
\hline Cosamaloapan & Gabino Barreda & $18.18987^{\circ} \mathrm{N}, 96.09536^{\circ} \mathrm{W}$ & LACM PC 2679 / este trabajo \\
\hline Cuichapa & La Providencia & $18.75558^{\circ} \mathrm{N}, 96.76908^{\circ} \mathrm{W}$ & LACM PC 2680-2681/ este trabajo \\
\hline Los Tuxtlas & Catemaco & $18.423^{\circ} \mathrm{N}, 95.116^{\circ} \mathrm{W}$ & González-Soriano et al. 1997 \\
\hline Minatitlán & ca. $1 \mathrm{~km} \mathrm{~N} \mathrm{de} \mathrm{Minatitlán}$ & $18.02777^{\circ} \mathrm{N}, 94.56694^{\circ} \mathrm{W}$ & Zamora-Abrego et al. 2006 \\
\hline Veracruz & Calzada Costa Verde & $19.16911^{\circ} \mathrm{N}, 96.11845^{\circ} \mathrm{W}$ & LACM PC 2668/ este trabajo \\
\hline Yanga & $2.5 \mathrm{~km}$ O de Yanga & $18.83632^{\circ} \mathrm{N}, 96.80701^{\circ} \mathrm{W}$ & Vásquez-Cruz et al., 2020 \\
\hline
\end{tabular}




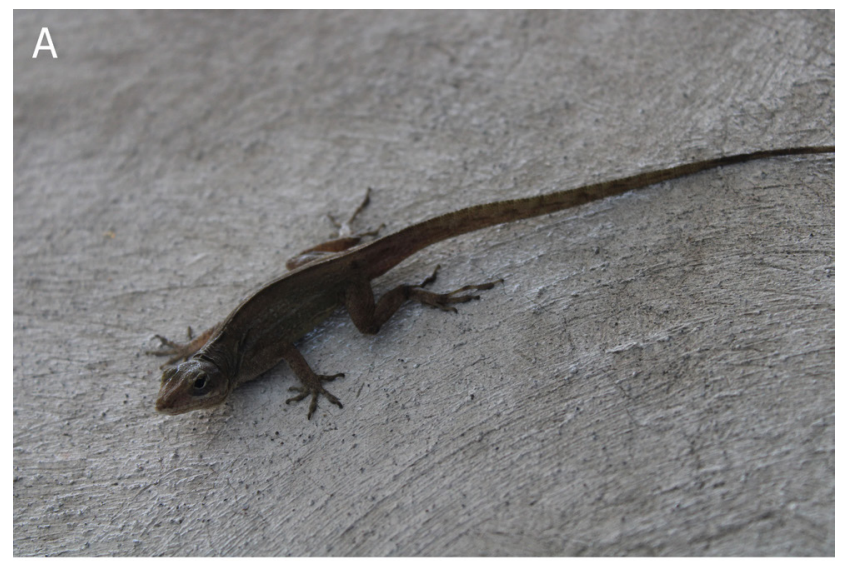

B

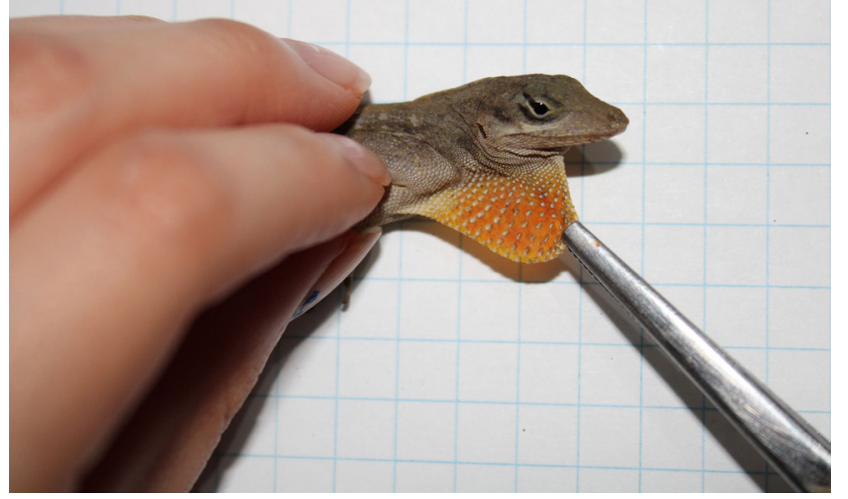

Figure 3. I(A) An individual of Anolis sagrei (LACM PC 2679) from the municipality of Cosamaloapan de Carpio, Veracruz, Mexico. (B) view of the gular fan.

Figura 3. (A) Un individuo de Anolis sagrei(LACM PC 2679) del municipio de Cosamaloapan de Carpio, Veracruz, México. (B) vista del abanico gular.

(Calzada Costa Verde) $\left(19.16911^{\circ} \mathrm{N}, 96.11845^{\circ} \mathrm{W}\right.$, WGS 84, elev. 10 m. s.n.m.). Estos individuos se encontraban exhibiendo comportamiento de flexión o push up y cambios de coloración de marrón a negro (aun visible el patrón), no hubo extensión de la gula, ya que se encontraban en eventos de combate agresivo entre los tres individuos (LACM PC 2668; Fig. 1). Este registro se encuentra a $3.5 \mathrm{~km} \mathrm{~N}$ (línea aérea) del registro previamente conocido más cercano en la colonia Las Vegas, en el municipio de Boca del Río (LACM PC 2471; Vásquez-Cruz et al., 2020).

El 10 de noviembre del 2020, alrededor de las 1400 horas, observamos al menos diez individuos adultos de la especie $A$. sagrei en el interior de un jardín entre plantas ornamentales cerca del ingenio azucarero en la localidad de Providencia, en el municipio de Cuichapa (18.75558 $\mathrm{N}, 96.76908^{\circ} \mathrm{O}$, WGS 84, elev. 395 m s.n.m.). De estas lagartijas se fotografiaron dos machos adultos (LACM PC 2680; LACM PC 2681; Fig. 2). Este registro se encuentra a $9.6 \mathrm{~km} \mathrm{~S}$ (línea aérea) del registro previamente conocido más cercano en la localidad de Yanga, en el Municipio de Yanga (LACM PC 2466; Vásquez-Cruz et al., 2020).

El 19 de noviembre del 2020, alrededor de las 1200 horas, observamos a 6 individuos adultos ( 2 machos y 4 hembras) en la localidad de Gabino Barreda en el municipio de Cosamaloapan de Carpio (18.1898 ${ }^{\circ} \mathrm{N}, 96.0953^{\circ}$ O, WGS 84, elev. 40 m s.n.m.) dentro de un vivero de plantas ornamentales. Este registro (LACM PC 2679; Fig. 3) se encuentra a 73 km SO (línea aérea) del registro previamente conocido más cercano en la localidad de Mandinga, en el Municipio de Alvarado (MZFC 27327; ToscanoFlores \& Calzada-Arciniega, 2015).

Si bien, los registros pertenecientes a los municipios de Cuichapa y Veracruz, se encuentran a poca distancia de los reportado en el municipio de Yanga y Boca del Río respectivamente, estos verifican la presencia de la especie en municipios cercanos y muy posiblemente por colonización natural progresiva como lo sugieren Vásquez-Cruz et al., (2020) o a través de plantas ornamentales, como lo han sugerido otros autores (e.g. Norval et al., 2002). En cuanto al establecimiento de la especie en la localidad de Gabino Barreda en el municipio de Cosamaloapan de Carpio, aparentemente ha sido reciente, ya que en visitas anteriores al vivero y alrededores en 2018 y 2019 no se observaron. En este último caso, las plantas ornamentales parecen ser la razón más obvia de la colonización.

La distribución de A. sagrei se extiende más hacia el centrooeste del estado (Fig. 4). El incremento de los registros de A. sagrei en un corto tiempo sugiere que su distribución en el Golfo de México es mayor de lo que se conoce y es posible que se esté extendiendo al interior del país y en la región norte del estado de Oaxaca que colinda con la localidad de Gabino Barrera en Veracruz. Es necesario realizar muestreos en localidades que colinden con las conocidas hasta el momento, lo que permitirá conocer la distribución actual de la especie y su posible impacto en las especies nativas.

Agradecimientos.- A María del Mar Rivera Zilli por proporcionar la foto de la observación, a Neftalí Camacho por catalogar las fotografías digitales. A dos revisores anónimos por sus valiosos comentarios.

\section{LITERATURA CITADA}

González Soriano E., R. Dirzo \& C. Vogt (Eds.). 1997. Historia Natural de Los Tuxtlas. UNAM, Instituto de Biología, México, D.F. 


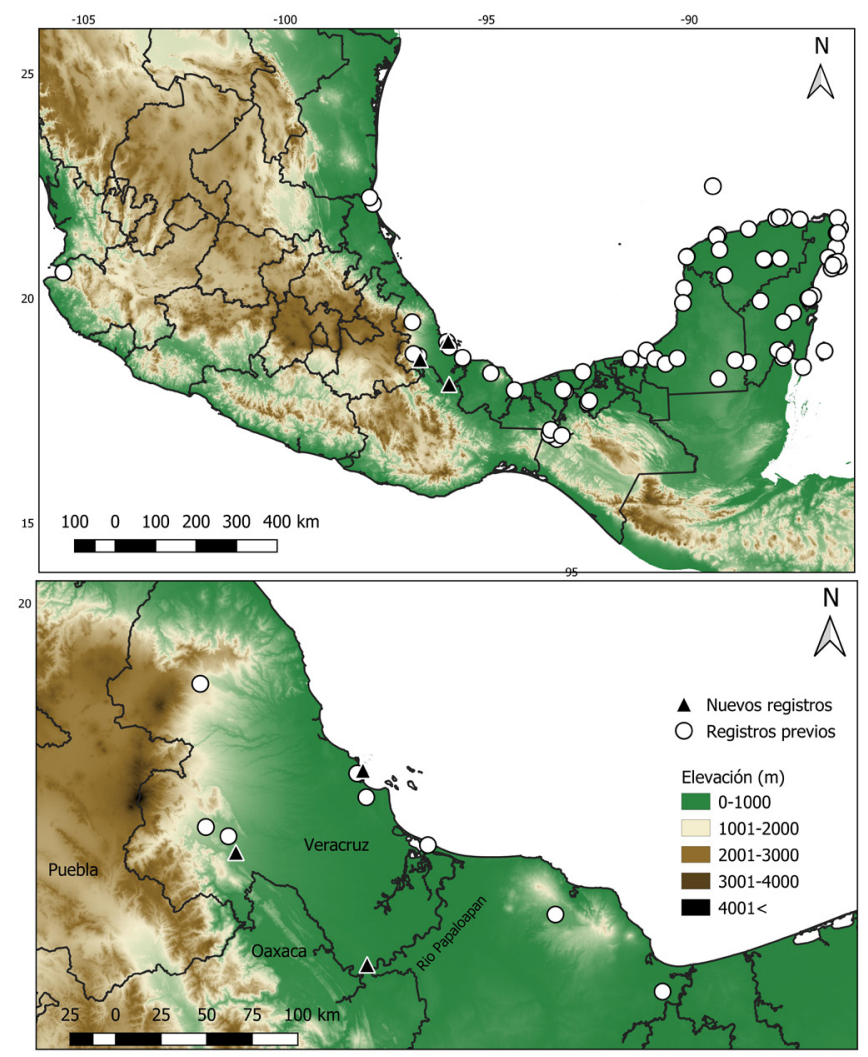

Figure 4. Map of Anolis sagrei distribution points in México and in the municipalities of state of Veracruz.

Figura 4. Mapa de puntos de registro de Anolis sagrei en México y en los municipios del Estado de Veracruz, México.
Köhler, G. 2008. Reptiles of Central America. 2nd edition. Herpeton Verlag, Offenbach, Germany.

Norval, G., J.J. Mao, H.P. Chu \& L.C. Chen. 2022. A New Record of an Introduced Species, the Brown Anole (Anolis sagrei) (Duméril \& Bibron, 1837), in Taiwan. Zoological Studies 41:332-336

Powell, R. \& R.W. Henderson. 2012. Island lists of West Indian amphibians and reptiles. Bulletin of the Florida Museum of Natural History 51:85-166.

Toscano-Flores, C. \& R.A. Calzada-Arciniega. 2015. Geographic distribution: Anolis sagrei (Brown Anole). Herpetological Review 46:215.

Vásquez-Cruz, V., A. Reynoso-Martínez, A. Fuentes-Moreno \& L. Canseco-Márquez. 2020. The Distribution of Cuban Brown Anoles, Anolis sagrei (Squamata: Dactyloidae), in Mexico, with New Records and Comments on Ecological Interactions. IRCF Reptiles \& Amphibians 27:29-35.

Venerozo-Tlazalo, D.G., R. Serna-Lagunes \& V. Vásquez-Cruz. 2017. Norops sagrei (Duméril \& Bibron, 1837). Mexico, Veracruz. Mesoamerican Herpetology 4:197-198.

Zamora-Abrego, J.G., U.O. García-Vázquez, A. Nieto-Montes de Oca \& L. Canseco-Márquez. 2006. Geographic distribution: Anolis sagrei (Brown Anole). Herpetological Review 37:493. 